\title{
Shear dispersion in the thermocline and the saline intrusion ${ }^{\text {is }}$
}

\author{
Hsien-Wang Ou ${ }^{\mathrm{a}, *}$, Xiaorui Guan ${ }^{\mathrm{b}}$, Dake Chen ${ }^{\mathrm{c}, \mathrm{d}}$ \\ a Division of Ocean and Climate Physics, Lamont-Doherty Earth Observatory, Columbia University, 61 Rt. 9W, Palisades, NY 10964, United States \\ ${ }^{\mathrm{b}}$ Consultancy Division, Fugro GEOS, 6100 Hillcroft, Houston, TX 77081, United States \\ ${ }^{c}$ Lamont-Doherty Earth Observatory, Columbia University, United States \\ ${ }^{\mathrm{d}}$ State Key Laboratory of Satellite Ocean Environment Dynamics, Hangzhou, China
}

\section{A R T I C L E I N F O}

\section{Article history:}

Received 11 March 2011

Received in revised form

15 March 2012

Accepted 19 March 2012

Keywords:

Saline intrusion

Shear dispersion

Lateral diffusion

Isopycnal diffusivity

Tracer dispersion

Thermocline intrusion

\begin{abstract}
A B S T R A C T
Over the mid-Atlantic shelf of the North America, there is a pronounced shoreward intrusion of the saltier slope water along the seasonal thermocline, whose genesis remains unexplained. Taking note of the observed broad-band baroclinic motion, we postulate that it may propel the saline intrusion via the shear dispersion. Through an analytical model, we first examine the shear-induced isopycnal diffusivity ("shear diffusivity" for short) associated with the monochromatic forcing, which underscores its varied even anti-diffusive short-term behavior and the ineffectiveness of the internal tides in driving the shear dispersion. We then derive the spectral representation of the long-term "canonical" shear diffusivity, which is found to be the baroclinic power band-passed by a diffusivity window in the log-frequency space. Since the baroclinic power spectrum typically plateaus in the low-frequency band spanned by the diffusivity window, canonical shear diffusivity is simply $1 / 8$ of this low-frequency plateau independent of the uncertain diapycnal diffusivity. Applied to the mid-Atlantic shelf, this canonical shear diffusivity is about $20 \mathrm{~m}^{2} \mathrm{~s}^{-1}$, which is sufficient to account for the observed tracer dispersion or saline intrusion in the thermocline.
\end{abstract}

(c) 2012 Elsevier Ltd. All rights reserved.

\section{Introduction}

Over the mid-Atlantic shelf of the North America, the summer hydrography is dominated by the seasonal thermocline caused by the surface heating. Since the thermal contrast dominates the density signature, the seasonal thermocline generally aligns with the pycnocline, along which there is little temperature variation. In contrast, the salinity varies discernibly along the pycnocline and, as its most striking feature, exhibits a shoreward intrusion of the saltier slope water. Because of its spatio-temporal variability, this subsurface salinity maximum may not be captured by individual soundings (Flagg et al., 1994; Lentz, 2003), but it is of sufficiently common occurrence to manifest prominently in the averaged cross-shore sections. An example of the latter representing an average of 12 transects in mid-September is reproduced in Fig. 1 (taken from Burrage and Garvine, 1988), which shows a distinct tongue of saltier slope water that has intruded a distance of $O(10 \mathrm{~km})$ along the thermocline. Using the observed temperature and salinity range of $10 \mathrm{~K}$ and 0.5 , respectively, across the saline intrusion, the density ratio is about $5-$ the reason that the feature does not significantly perturb the density field hence may be regarded as dynamically passive.

\footnotetext{
Lamont-Doherty Earth Observatory Contribution Number 7557.

* Corresponding author. Tel.: +1845 365 8338; fax: +1845 3658157.

E-mail address: dou@ldeo.columbia.edu (H.-W. Ou).
}

With the abatement of wind in summer, Houghton and Marra (1983) suggested that the saline intrusion constitutes the primary exchange process between the shelf and slope waters in the stratified season. Quantitatively, Gordon and Aikman (1981) estimated that the saline intrusion provides up to half the salt needed to balance the riverine discharge; and based on his census of soundings in the Mid-Atlantic Bight, Lentz (2003) calculated that the shelf salinity would be 0.3 lower without the saline intrusion, a significant fraction of the total salinity range across the shelf. Even taking into account considerable uncertainty in these estimates, the importance of the saline intrusion to the salt balance of the shelf water is palpable; the impetus to understand its genesis thus extends beyond mere dynamical curiosity.

Several causes have been conjectured for the saline intrusion, none however are strongly argued or computationally demonstrated. Boicourt and Hacker (1976) and Flagg et al. (1994) opined that the feature could be induced by wind, a linkage however unsupported by observational evidence (Burrage and Garvine, 1988; Flagg et al., 1994; Lentz, 2003; Churchill et al., 2003). Welch (1981) and Aikman (1984) suggested that it is driven by the lateral pressure gradient, but the latter is coupled to the motion field hence may not be regarded as an independent forcing. Some have postulated its generation by the double-diffusive interleaving (Horne, 1978; Gordon and Aikman, 1981; Houghton and Marra, 1983), but the requisite concurrent thermal and saline gradient on density surfaces is not apparent (see Fig. 1), and the 


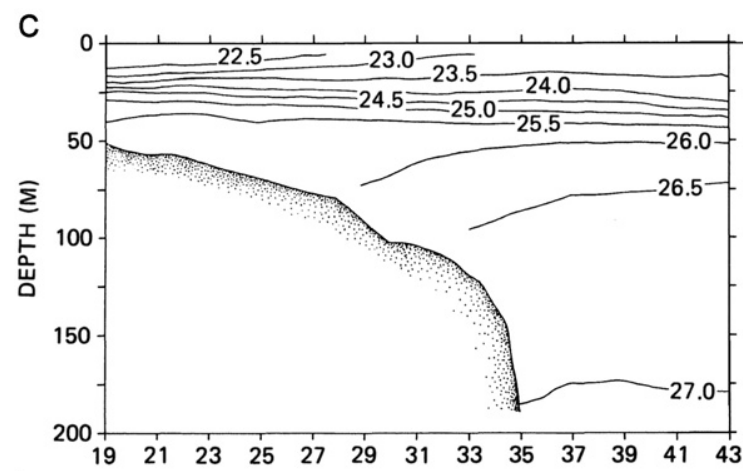

b
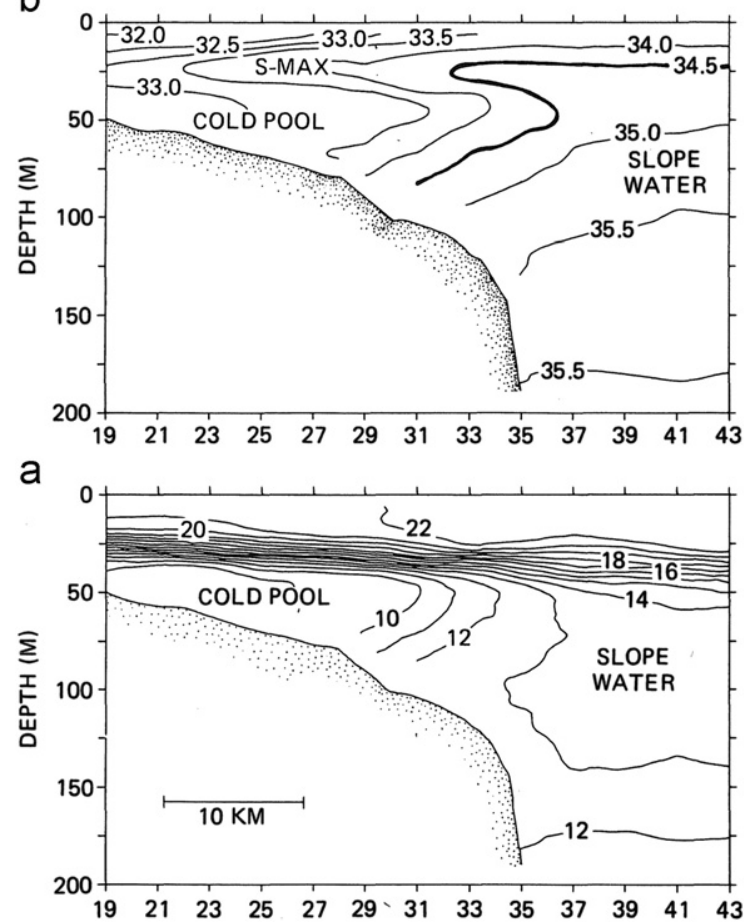

Fig. 1. The cross-shore hydrography in the Mid-Atlantic Bight in mid-September (averaged over 12 transects) for temperature (a), salinity (b), and density (c). The 34.5 isohaline marks the boundary of the slope water, which has intruded a distance of $O(10 \mathrm{~km})$ onto the shelf along the seasonal thermocline (taken from Burrage and Garvine, 1988).

calculated growth rate in any event seems too small (Lentz, 2003). Although the frontal instability enhanced by impinging Gulf Stream eddies may inject the slope water onto the shelf (Churchill et al., 2003), there is no reason for it to be constricted to the depth range of the thermocline. Nonlinear rectification by the baroclinic tides could drive an onshore flow in the thermocline (Ou and Maas, 1986), it is however an Eulerian-mean that may not transport passive tracers. To recap, despite many propositions, the genesis of the saline intrusion remains unresolved, which points to a significant gap in our understanding of the shelf-slope exchange.

\section{Hypothesis}

In seeking a possible explanation of the saline intrusion, we take note of the observed prominence of internal tides on the shelf. They are generated when the surface tides interact with the topographic slope and density stratification (Baines, 1982), which if super-inertial would propagate onto the shelf - sometimes in the form of solibores whose surface imprints can be seen in satellite images (Liu et al., 1998). Moored measurements (Colosi et al., 2001; MacKinnon and Gregg, 2003a) showed additionally that the baroclinic current may reach several tens of $\mathrm{cm} \mathrm{s}^{-1}$, but unlike its barotropic counterpart, the baroclinic power spectrum is broadband and contains comparable low-frequency as the tidal energy (see Fig. 2, adapted from Sharples et al., 2001), a property of particular significance in our discussion.

Since the baroclinic shear is concentrated in the thermocline (MacKinnon and Gregg, 2003a; Palmer et al., 2008), so is the diapycnal mixing induced by the shear instability, and indeed at the passage of the solibores the diapycnal diffusivity may be elevated by two orders of magnitude (MacKinnon and Gregg, 2003b; Moum et al., 2003). With the concurrent vertical shear and diapycnal mixing in the thermocline, one naturally wonders if the well-known mechanism of shear dispersion may drive the observed saline intrusion, the question that motivated the present study.

Besides the naturally occurring saline intrusion, the tracer release experiments also showed enhanced lateral dispersion in the thermocline with an effective diffusivity of $O\left(10 \mathrm{~m}^{2} \mathrm{~s}^{-1}\right)$ (Houghton, 1997; Sundermeyer and Ledwell, 2001; Ledwell et al., 2004). To uncover the source of this dispersal, Sundermeyer and Ledwell (2001) carried out numerical integrations of the tracer distribution using the observed shear and diapycnal diffusivity, and found that the calculated lateral diffusivity is only a fraction of that required. Because of this discrepancy, they suspected that there could be episodic mixing events within the thermocline that might not be captured by the measured vertical diffusivity. This is plausible since the latter is based on the vertical spread of the tracer patch hence indicative of the background diffusivity - not the diffusivity within the tracer patch that facilitates the shear dispersion. Additionally, they postulated that the geostrophic adjustment accompanying such vertical mixing events may account for the observed lateral diffusion (Sundermeyer et al., 2005) - a proposition not yet substantiated (Sundermeyer and Lelong, 2005). Nonetheless, since such vertical mixing events would augment the diapycnal diffusivity, it may partially bridge the above shortfall.

Following Taylor's (1953) consideration of the shear dispersion in a steady flow, the study of the mechanism has been extended

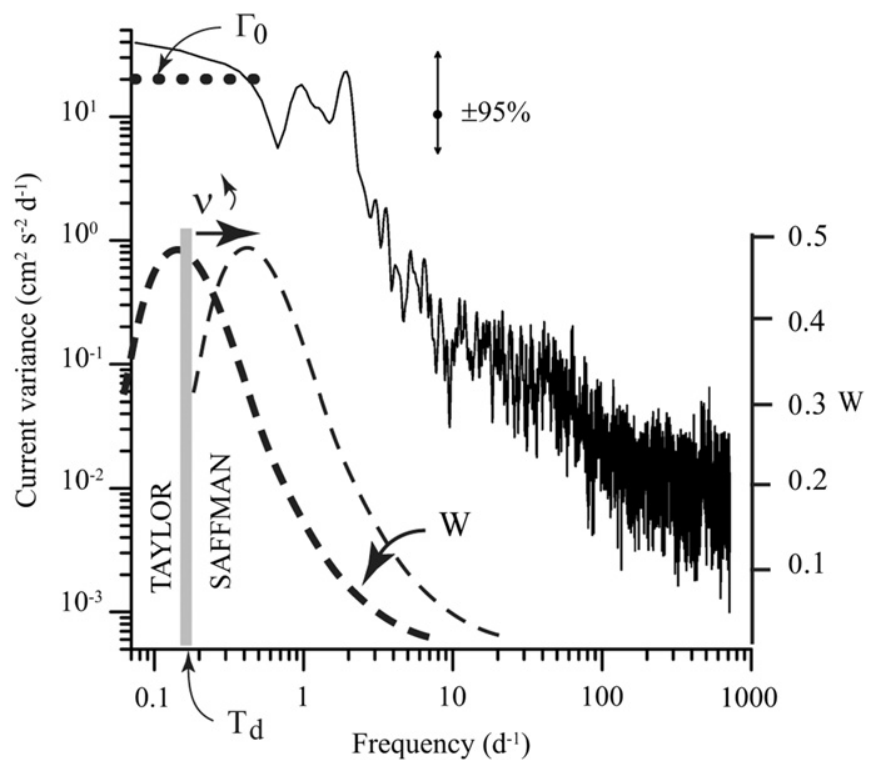

Fig. 2. Diffusivity windows $(W)$ superimposed on the baroclinic power spectrum of Sharples et al. (2001) (thick-dashed for the observed vertical diffusivity $v$ and thin-dashed when $v$ is doubled). The windows peak at the diffusive time $\left(T_{\mathrm{d}}\right)$ separating the Taylor and Saffman regimes. The dotted line marks the lowfrequency plateau of the power density. 
to a flow that is oscillatory in time to address the tidal dispersal of tracers (Okubo, 1967; Zimmerman, 1986) and to a flow that is sinusoidal in the vertical as well to examine its driving by the internal waves (Young et al., 1982). Since our inquiry is the shear dispersion in the thermocline where the current shear is concentrated, it affords certain simplification in the model formulation when compared with Young et al. (1982); on the other hand, since the baroclinic motion is inherently broadband — unlike the sharply peaked barotropic tides, one needs to take a spectral view of the process unnecessary for the aforementioned studies.

The remainder of the paper is organized as follows. We formulate the model in Section 3 and examine the shear diffusivity induced by monochromatic forcing in Section 4. We derive the spectral representation of the shear diffusivity in Section 5 and carry out a numerical test of the analytical formula in Section 6 . We apply the model results to the shelf environment in Section 7 and show that the shear diffusivity is sufficient to account for the observed tracer dispersion and saline intrusion in the thermocline. We summarize the main findings and provide additional discussion in Section 8.

\section{Model formulation}

To elucidate the essential physics, it suffices to consider a level pycnocline (used interchangeably with thermocline) embedded in cross-shore baroclinic current as shown in Fig. 3. The current is oscillatory in time, unvarying laterally, and its shear confined to the pycnocline; this shear would induce diapycnal diffusivity through shear instability, which thus is similarly confined. We recognize that the observed thermocline undulates due to the semi-diurnal tides, which being super-inertial are progressive, but since we are concerned with shear dispersion over much longer timescale, we shall take the model thermocline to be the tidalmean hence more aptly the "thermal band" spanned by the undulation. One is also aware of the strong vertical mixing near the ocean surface and bottom due respectively to the wind- and friction-induced shear, which however plays no part in the shear dispersion in the thermocline. The assumed confinement of the vertical diffusivity to the thermocline pertains to its contrast from the smaller background mid-column value, as seen in the observed profiles (for example, MacKinnon and Gregg, 2003b, their Fig. 11), which may also be justified by the slow leakage of the tracer released in the thermocline (Ledwell et al., 1993). While the observed vertical mixing within the thermocline is highly episodic and its varied phasing with the current shear may introduce considerable complication, we shall nonetheless for simplicity assign a constant vertical diffusivity to the pycnocline representing some proper space-time average. This assumption can be partially justified a posteriori by the dominance of the

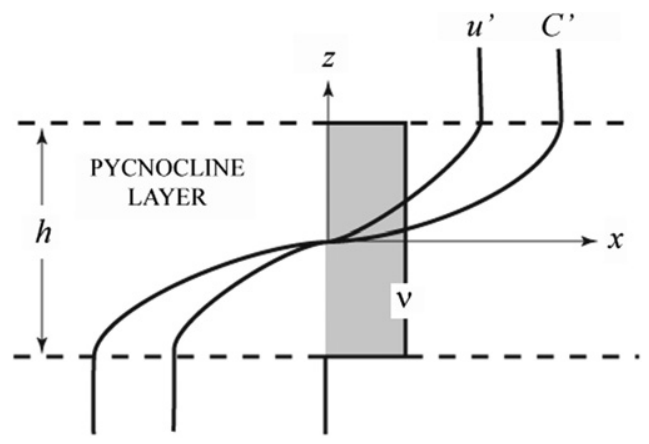

Fig. 3. The model configuration of a level pycnocline of thickness $h$ and uniform vertical diffusivity $v$ (zero outside) together with profiles of the baroclinic current $u^{\prime}$ and the tracer perturbation $C^{\prime}$. low-frequency motion in driving the long-term shear dispersion, which of particular significance has ameliorated its dependence on the vertical diffusivity (Section 7).

We consider the tracer concentration $C$ inside the pycnocline governed by the equation

$C_{t}+u^{\prime} C_{x}=v C_{z z}$,

where the subscripts indicate partial derivatives ( $x$ and $z$ are in the cross-shore and up directions), $u^{\prime}$ is the baroclinic oscillatory current and $v$, the vertical diffusivity. The neglect of ambient vertical diffusivity implies zero tracer flux out of the pycnocline so the concentration $C$ has zero normal-gradient at the layer boundary. Although one may formulate a more precise boundary condition with non-zero outer vertical diffusivity, this would only complicate the mathematics but not alter the essential physics. To proceed, we decompose the concentration in the pycnocline into its vertical average through the layer (angle-bracketed) and deviation (primed):

$C=\langle C\rangle+C^{\prime}$

Taking the vertical average of (1) and applying the zero normal-gradient boundary condition, we obtain, noting that $u^{\prime}$ is assumed laterally uniform,

$\langle C\rangle_{t}+\left\langle u^{\prime} C^{\prime}\right\rangle_{x}=0$,

which can be subtracted from (1) to yield

$C_{t}^{\prime}+u^{\prime}\langle C\rangle_{x}+\left(u^{\prime} C^{\prime}\right)_{x}-\left\langle u^{\prime} C^{\prime}\right\rangle_{x}=v C_{z z}^{\prime}$.

If we assume the motion to be sufficiently weak that the quadratic terms can be neglected, the above equation is simplified to

$C_{t}^{\prime}+u^{\prime}\langle C\rangle_{x}=v C_{z z}^{\prime}$

an approximation to be assessed a posteriori (see later discussion). Defining the "tracer" displacement $\varsigma^{\prime}$ via

$C^{\prime} \equiv-\varsigma^{\prime}\langle C\rangle_{x}$,

it then satisfies the equation

$\varsigma_{t}^{\prime}-v \varsigma_{z z}^{\prime}=u^{\prime}$,

hence is decoupled from the mean tracer field. One may remove the vertical-derivative terms by taking the current profile to be a halfsine function in the pycnocline (see Fig. 3 )

$u^{\prime}=\hat{u}(t) \sin (\pi z / h)$

where $h$ is the pycnocline thickness and $z=0$ is set at its mid-point. Since this half-sine function is an eigenmode of the tracer concentration as well, the solution to (7) is of the same form

$\varsigma^{\prime}=\hat{\varsigma}(t) \sin (\pi z / h)$

with the temporal part satisfying the equation

$\hat{\varsigma}_{t}+\alpha \hat{\varsigma}=\hat{u}$,

where

$\alpha \equiv v(\pi / h)^{2}$

is a parameter measuring the importance of the vertical mixing. Defining the "diffusive" time as

$T_{\mathrm{d}} \equiv 2 \pi / \alpha=(2 / \pi) h^{2} / v$,

and let $T_{\mathrm{f}}$ be the "forcing" period and $l_{\mathrm{e}}$, the particle excursion, then (10) implies a scale relation (brackets for scales),

$[\varsigma] \leq l_{\mathrm{e}}\left(1, T_{\mathrm{d}} / T_{\mathrm{f}}\right)$,

or the tracer displacement is bounded above by the excursion and can be considerably smaller if the vertical mixing is strong (that is, the diffusive time is short compared with the forcing period).

With (13), one may now reassess the neglect of the quadratic terms in (4). By comparing them with the second term, it requires that the tracer displacement be small compared with the 
horizontal scale characterizing the mean concentration, and based on (13), a sufficient condition would be the excursion itself satisfies this requirement. On the other hand, even if these conditions are not strictly met, we do not expect the quadratic terms to alter the essential results, as attested by their validation by primitive-equation calculations (Section 6).

With the above formulation, one may solve for the perturbation (10), which is phase-shifted by the vertical mixing. The resulting rectified flux then drives the evolution of the vertical-mean concentration via (3) - the essence of the shear dispersion. The derivation however can be facilitated by the introduction of the "shear" diffusivity that encodes the rectified flux, as discussed next.

\section{Shear diffusivity}

The rectified flux in (3) can be expressed in terms of the tracer displacement (6)

$\left\langle u^{\prime} C^{\prime}\right\rangle=-\left\langle u^{\prime} \varsigma^{\prime}\right\rangle \cdot\langle C\rangle_{x}$,

which allows the definition of the shear diffusivity (a vertically averaged property within the pycnocline) as

$k(t) \equiv\left\langle u^{\prime} \varsigma^{\prime}\right\rangle=\hat{u} \hat{\varsigma} / 2$

on account of (8) and (9). Integrating (10) from the time of the tracer release, we obtain the tracer displacement

$\hat{\zeta}=\int_{0}^{t} \hat{u}\left(t^{\prime}\right) e^{-\alpha\left(t-t^{\prime}\right)} \mathrm{d} t^{\prime}$,

which can be substituted into (15) to yield the (instantaneous) shear diffusivity. As such, it is seen that the shear diffusivity is a property only of the motion field hence laterally unvarying as well. As its auxiliary properties, we define the time-mean shear diffusivity (overbarred) as its average over the interval $[0, t]$ :

$\bar{k}(t) \equiv \frac{1}{t} \int_{0}^{t} k \mathrm{~d} t^{\prime}$,

and the time-integrated shear diffusivity (the suffix "int") as its integration over the same interval:

$k_{\text {int }}(t) \equiv \int_{0}^{t} k \mathrm{~d} t^{\prime}=\bar{k} t$.

Using the shear diffusivity, the time progression of the (vertical) mean concentration (3) becomes

$\langle C\rangle_{t}=k\langle C\rangle_{x x}$.

It is seen from this equation that if the concentration were initially Gaussian in $x$, it would remain so as time progresses with its second moment $l^{2}$ given by

$l^{2}=l_{0}^{2}+2 k_{\text {int }}$,

where the subscript " 0 " denotes the initial value.

Analytical expressions of the shear diffusivity and its auxiliary properties are given in Appendix for the monochromatic forcing, and some these are plotted in Fig. 4 for selected forcing periods and phases. The shear diffusivity in this figure has been nondimensionalized by the diffusivity scale

$[k] \equiv U^{2} / \alpha=U^{2} T_{\mathrm{d}} /(2 \pi)$,

where $U$ is the amplitude of the current difference across the pycnocline (referred as the "baroclinic amplitude") and time $t$ and the forcing period $T_{\mathrm{f}}$ have been non-dimensionalized by the diffusive time (12) so that

$\tau \equiv T_{\mathrm{f}} / T_{\mathrm{d}}=(2 \pi \omega)^{-1} \alpha$

with $\omega$ being the forcing frequency (cycles per unit time). The phase refers to the lag of the tracer release following the peak

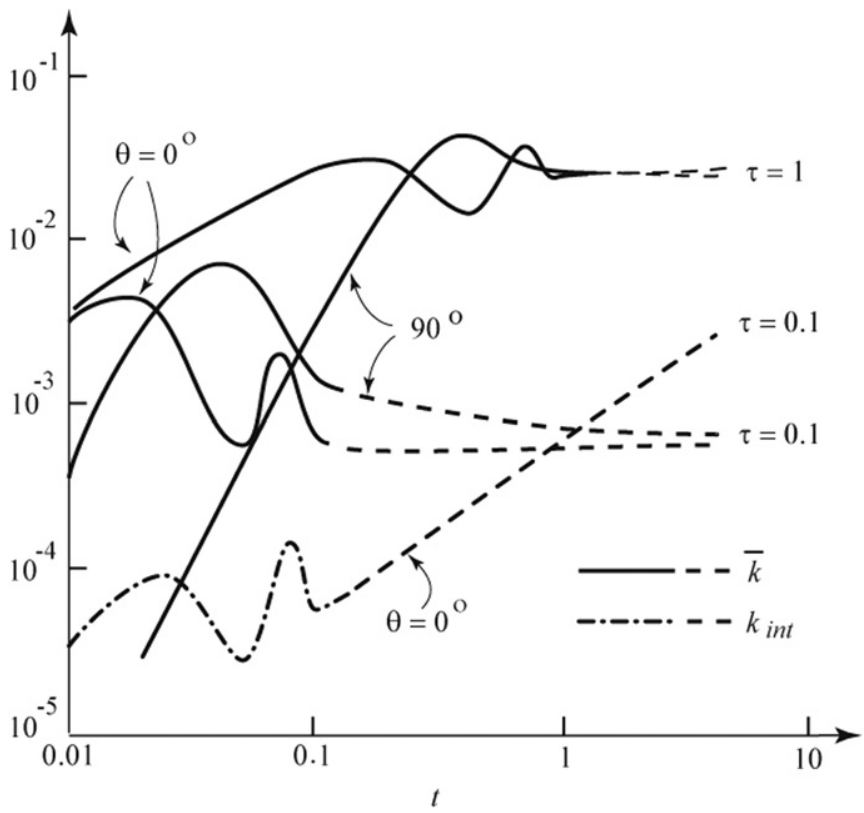

Fig. 4. Time progression of the non-dimensionalized shear diffusivity for selected forcing periods $\tau$ and phases $\theta$ (the curves are the same for phase increment of half cycles). Solid and dash-dotted lines are respectively the time-mean and timeintegrated value over the internal $[0, t]$ and their dashed extensions have been smoothed over the rapidly shortening wiggles (because of the log-time scale). The time and forcing period have been non-dimensionalized by the diffusive time and the phase pertains to the lag of the tracer release with respect to the peak positive baroclinic current.

positive baroclinic current (noting that since the shear diffusivity is quadratic in the forcing, the curves are the same for phase increment of half-cycles). It is seen that the time-mean shear diffusivity varies strongly in time, particularly during the first forcing cycle, which moreover is sensitive to the precise time of the tracer release. In fact, the instantaneous shear diffusivity could be negative during the sharp downturn of the time-mean an anti-diffusive behavior well recognized in the literature (Smith, 1982). On the other hand, the time-mean is always positive, so the tracer patch at any time has grown from its initial size.

As seen in Appendix, the time-mean shear diffusivity tends over large time to an "equilibrium" value of

$\bar{k} \rightarrow k_{\mathrm{eq}}=\frac{1}{16} \cdot \frac{\tau^{2}}{1+\tau^{2}} \quad$ at $\quad t \rightarrow \infty$,

which is a function only of the scaled forcing period $\tau$, and the large time is with respect to both the forcing period and the diffusive time. It is seen from (23) that the equilibrium shear diffusivity is greater for longer-period forcing (that is, greater $\tau$ ) and asymptotes to $1 / 16$. Qualitatively, the longer-period forcing induces greater perturbation in the tracer concentration, which is also more in phase with the baroclinic motion - both enhancing the shear dispersion; and for sufficiently long forcing period, the tracer concentration is in approximate steady-state diffusive balance with the forcing hence its amplitude as well as the vertically averaged flux no longer depends on the forcing period - the reason for the above asymptote. Shortening the forcing period, on the other hand, this equilibrium value (23) would decreases sharply when the forcing period drops below the diffusive time.

We have also plotted in Fig. 4 the time-integrated shear diffusivity (dash-dotted line), which would track the second moment of the tracer patch (20). At large time, the second moment grows linearly at 
a rate given by the equilibrium shear diffusivity (see (18) and (20)), but over the short term, given possible contractions, the tracer evolution provides a poor indicator of the equilibrium shear diffusivity. Since for small perturbations, the rectified flux and hence the shear diffusivity are additive among forcings, the above problem would be compounded by the presence of low-frequency forcing as the tracer patch would vacillate more widely and over longer stretch of time.

Since the equilibrium shear diffusivity is greater for lowerfrequency forcing, the above transient behavior may partly explain the observed increase of the lateral diffusivity over greater spatial (Ledwell et al., 1998) - and hence temporal scale. That is, as the time progresses, the tracer patch is increasingly subjected to lower-frequency forcing that drives more efficient shear dispersion. But despite the contractions of the tracer patch, at no time would it return to the initial size: there could be no spontaneous emergence of the tracer front.

\section{Spectral representation}

Despite the short-term variation in the shear diffusivity, one may nonetheless inquire about its hypothetical value when averaged over infinitely long time, corresponding to full spectral integration of the equilibrium value. This will be referred as the "canonical" shear diffusivity $\left(k_{\text {can }}\right)$, a property hence only of the baroclinic forcing and mixing environment. Extending (17) to infinite time, we have, using (15),

$k_{\mathrm{can}} \equiv \lim _{T \rightarrow \infty} \frac{1}{2 T} \int_{-T / 2}^{T / 2} \hat{u}(t) \hat{\zeta}(t) \mathrm{d} t$.

Substituting from (16) and expressing the baroclinic current in its Fourier coefficients so that

$\hat{u}(t)=\sum_{n=-\infty}^{n=\infty} \hat{u}_{n} e^{i 2 \pi n t / T}$,

with

$\hat{u}_{n} \equiv \frac{1}{T} \int_{-T / 2}^{T / 2} \hat{u}(t) e^{-i 2 \pi n t / T} \mathrm{~d} t$,

we derive that

$$
\begin{aligned}
k_{\text {can }} & =\lim _{T \rightarrow \infty} \frac{1}{2 T} \int_{-T / 2}^{T / 2} \hat{u}(t) \int_{-T / 2}^{t} \hat{u}\left(t^{\prime}\right) e^{-\alpha\left(t-t^{\prime}\right)} \mathrm{d} t^{\prime} \mathrm{d} t \\
& =\lim _{T \rightarrow \infty} \frac{1}{2 T} \int_{-T / 2}^{T / 2} \hat{u}(t) \int_{-T / 2}^{t} \sum_{n=-\infty}^{n=\infty} \hat{u}_{n} e^{i 2 \pi n t^{\prime} / T} e^{-\alpha\left(t-t^{\prime}\right)} \mathrm{d} t^{\prime} \mathrm{d} t \\
& =\lim _{T \rightarrow \infty} \frac{1}{2 T} \int_{-T / 2}^{T / 2} \hat{u}(t) \sum_{n=-\infty}^{n=\infty} \hat{u}_{n} \frac{1}{i 2 \pi n / T+\alpha} \cdot e^{i 2 \pi n t / T} \mathrm{~d} t \\
& =\lim _{T \rightarrow \infty} \frac{1}{2} \sum_{n=-\infty}^{n=\infty} \frac{\hat{u}_{n}}{i 2 \pi n / T+\alpha} \cdot \frac{1}{T} \int_{-T / 2}^{T / 2} \hat{u}(t) e^{i 2 \pi n t / T} \mathrm{~d} t \quad \text { rearrange } \\
& =\lim _{T \rightarrow \infty} \frac{1}{2} \sum_{n=-\infty}^{n=\infty} \frac{\hat{u}_{n} \hat{u}_{n}^{*} T}{i 2 \pi n / T+\alpha} \cdot \frac{1}{T}
\end{aligned}
$$

With $1 / T$ tending to $\mathrm{d} \sigma$ and $n / T$ to $\sigma$ in the limit, (27) becomes

$$
\begin{aligned}
k_{\text {can }} & =\frac{1}{2} \int_{-\infty}^{\infty} \frac{F(\sigma)}{i 2 \pi \sigma+\alpha} \mathrm{d} \sigma \\
& =\frac{1}{4 \pi} \int_{0}^{\infty} \Gamma(\sigma) W(\sigma) \mathrm{d}(\ln \sigma),
\end{aligned}
$$

where

$$
F(\sigma) \equiv \lim _{T \rightarrow \infty}\left|\hat{u}_{n}\right|^{2} T
$$

is the baroclinic power spectrum,

$\Gamma(\sigma) \equiv 2 F(\sigma)$ for $\sigma>0$, its one-sided counterpart (corresponding to the observed spectrum shown in Fig. 2) and

$W(\sigma) \equiv \frac{\tau}{1+\tau^{2}}$,

with $\tau$ defined in (22) but generalized to the Fourier frequency, will be referred as the diffusivity window.

To check that (28) would reduce to the equilibrium shear diffusivity for monochromatic forcing (with frequency $\omega$ ), one notes that such forcing has a power spectrum of (see also Jenkins and Watts, 1968, Section 6.2.2)

$\Gamma(\sigma)=\frac{1}{2}\left(\frac{U}{2}\right)^{2} \delta(\omega-\sigma)$,

which when substituted into (28) yields indeed

$k_{\text {can }}=\frac{U^{2}}{\alpha} \cdot \frac{\tau^{2}}{16\left(1+\tau^{2}\right)}=[k] k_{\mathrm{eq}}$

on account of the definitions (21)-(23).

Based on (28), the canonical shear diffusivity is simply the baroclinic power band-passed in the log-frequency space by the diffusivity window (the thick dashed curve in Fig. 2) - the latter a function only of the diffusive time. In contrast to the scale definition of the shear diffusivity (21) motivated by comparing the efficiency of different forcing frequency (hence of the same amplitude and vertical diffusivity), here in the spectral space, we separate the shear diffusivity into its two controlling elements: the forcing defined by the baroclinic power spectrum $\Gamma(\sigma)$ and the vertical mixing encapsulated in the diffusivity window $W(\sigma)$. The separation allows the above spectral interpretation of the shear diffusivity, which moreover facilitates ready comparison between different forcing and mixing environments.

Because of the use of the log-frequency scale, varying the vertical diffusivity merely displaces the diffusivity window but leaves its shape unchanged (as seen in Fig. 2). And since the diffusivity window is invariant to the transformation: $\tau \rightarrow 1 / \tau$, it is symmetric about its peak located at the diffusive time. Increasing the vertical diffusivity, for example, would move the window to the right (the thin dashed line), so for a monochromatic forcing lying to the left of the peak, the shear diffusivity would weaken while the opposite is true for a forcing lying to the right; the disparate dependence corresponds respectively to the wellknown Taylor (1953) and Saffman (1962) regimes. One should note that although the diffusivity window drops off toward the low frequency, it is compensated by the expanding unit frequency interval in the log-frequency space, so given the same power, the low-frequency forcing contributes more strongly to the canonical shear diffusivity, which is thus entirely consistent with the earlier deduction that the equilibrium shear diffusivity asymptotes in the low-frequency limit (Section 4).

\section{Numerical test}

Since the analytical model is highly idealized, including the linearized Eq. (5) governing the perturbation, it is desirable if we can test the diffusivity window from numerical calculations using a primitive-equation model. The model we use is a free-surface, $z$-coordinate model originally developed by Wang (1982), which has been validated by its use in many coastal studies (e.g. Chen et al., 2003), and although it is relatively unsophisticated comparing with some other models, it suffices for our purpose.

From the numerical solution, we shall diagnose the shear diffusivity $k_{\text {eq }}^{*}$ (starred for dimensional value), which is then converted to the diffusivity window via (see [23] and [31])

$W(\tau)=\frac{16}{[k] \tau} \cdot k_{e q}^{*}$ 
and compared with the analytical formula (31). The conversion involves four parameters: the baroclinic amplitude $U$, the thermocline thickness $h$, the vertical diffusivity $v$ and the forcing period $T_{\mathrm{f}}$ (see definitions [12], [21] and [22]); and to have a better control of these "external" parameters, they will be directly imposed. Specifically, we configure the model on a flat-bottom cross-shore domain of depth $100 \mathrm{~m}$ and width $60 \mathrm{~km}$ with the corresponding grid spacing of $4 \mathrm{~m}$ and $1 \mathrm{~km}$; we impose an initial temperature field that varies only in the vertical, increasing linearly from 10 to $20{ }^{\circ} \mathrm{C}$ through a mid-depth thermocline layer (homogenous outside the layer); we assign a constant vertical diffusivity in this layer (zero outside); we prescribe the oscillatory baroclinic current at the offshore boundary, which has uniform shear in the thermocline layer (zero shear outside); and we apply the radiative condition at the inshore boundary. We set as a control case the following values: $U=0.2 \mathrm{~m} \mathrm{~s}^{-1}, h=16 \mathrm{~m}, v=10^{-3} \mathrm{~m}^{2} \mathrm{~s}^{-1}$ and $T_{\mathrm{f}}=0.5 \mathrm{~d}$; these and other values used below are chosen to span a sufficient range to test the analytical formula, so they need not be realistic.

We use the salinity as a proxy for the passive tracer (that is, turning off its effect on the density) and release at $t=0$ a $1 \mathrm{~km}-$ wide salinity strip (of value 35 against the background value of 33, see Fig. 5) and examine its subsequent evolution. Although there could be initial vacillation in the vertical-mean salinity as expected from Fig. 4, the large uncertainty in calculating this mean during the initial period and the need to resolve distance much finer than the excursion (about $0.7 \mathrm{~km}$ for the control case) do not allow such simulations. On the other hand, the primary outcome of the analytical derivation is the equilibrium shear diffusivity, which can be adequately tested if the integration time is sufficiently long that the growth of the tracer patch has stabilized. For the control case of forcing period $0.5 \mathrm{~d}$ and an estimated diffusive time of $1.9 \mathrm{~d}$, this stabilization occurs after a few days, and the numerical patches are plotted in Fig. 5 for $t=0$, 5 and $10 \mathrm{~d}$. It is seen that because of the near-zero background vertical diffusivity, there is little diffusive loss of the tracer from the thermocline (that is, the areas under the curves are largely preserved), hence duplicating the boundary condition for the tracer in the analytical model. With the absence of the lateral diffusivity in the model, the growth of the tracer patch in the

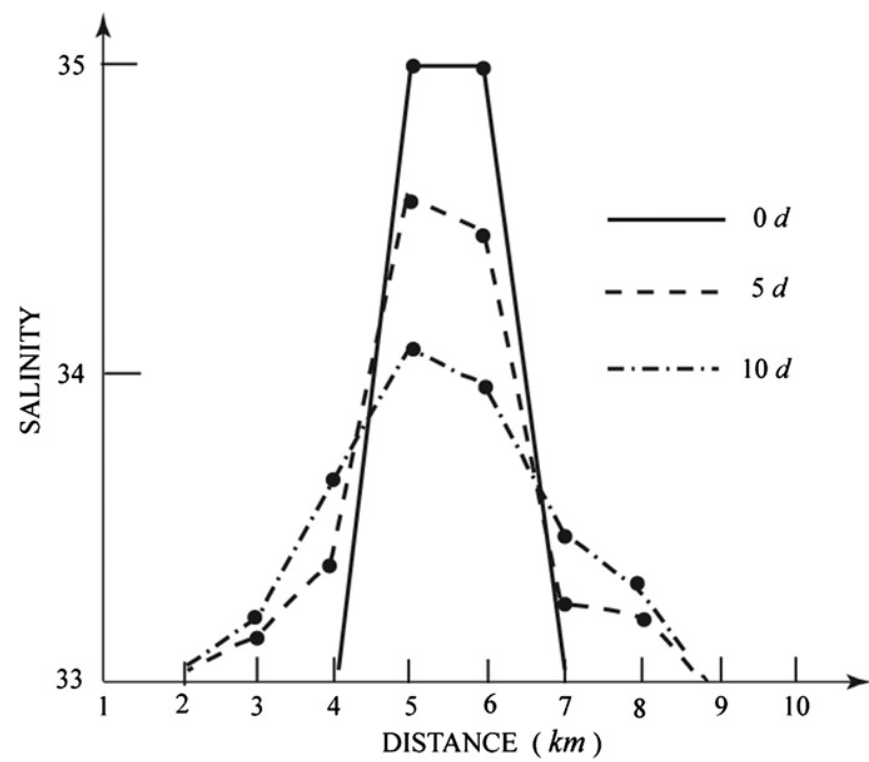

Fig. 5. The salinity patch in the thermocline plotted against the cross-shore distance for the control case. The solid, dashed and dash-dotted lines are for $t=0$, 5 and $10 \mathrm{~d}$, respectively. thermocline is due solely to the shear dispersion, which thus offers a qualitative demonstration of the process.

For quantitative comparison, we shall diagnose the (equilibrium) shear diffusivity from the numerical solution by taking it to be the average of $(2 t)^{-1}\left(l^{2}-l_{0}^{2}\right)$ between 5 and 10 forcing cycles (see (18) and (20)), and we assign as its error the standard deviation during the same duration. Since the baroclinic amplitude enters the shear diffusivity only through the quadratic scaling (21), this dependence will be first tested by plotting the diagnosed shear diffusivity against the baroclinic amplitude, as shown in Fig. 6. The numerical data points are in circles with the open circle marking the control case (the errors are too small to be shown), and for comparison a curve representing the quadratic dependence is drawn through the control case. It is seen that the numerical points generally follow the curve, in support of the quadratic scaling.

With the baroclinic amplitude fixed at its control value, we then vary other three parameters and the diffusivity window diagnosed from the numerical solution (34) is plotted against the scaled forcing period (22) in Fig. 7. The open circle marks the control case and, moving from left to right, the solid circles are for the additional thermocline thickness of 24 and $12 \mathrm{~m}$, the solid squares are for the additional forcing periods of 1 and $2 \mathrm{~d}$, and the crosses mark the three forcing periods for vertical diffusivity of $5 \times 10^{-3} \mathrm{~m}^{2} \mathrm{~s}^{-1}$ (five times the control value). The standard deviations are also indicated, which generally are greater for longer time integrations due perhaps to the cumulative drift of the base state. For comparison, the solid curve is the modelderived diffusivity window (31), and it is seen that the numerical points have captured the general shape of the analytical curve, particularly the peak at the diffusive time $(\tau=1)$ that divides the Saffman and Taylor's regimes (Section 5).

We noticed a slight but systematic shift of the numerical points to higher $\tau$, which could be attributed to the linear current profile used in the thermocline. To approximate this profile by the halfsine function used in the analytical model implies a greater thermocline thickness hence smaller vertical diffusivity than that imposed in the numerical solution. As such, the actual diffusive time is underestimated, which may account for the above shift. Otherwise, the numerical values compare favorably with the analytical curve - even though the neglect of the quadratic terms in (5) cannot be strictly justified in some cases (for example, the tracer displacement is greater than $1 \mathrm{~km}$ for the two solid squares hence not small compared with the lateral scale of the mean concentration). This quantitative comparison suggests that the analytical formula of the shear diffusivity is more widely applicable than some the model assumptions would indicate.

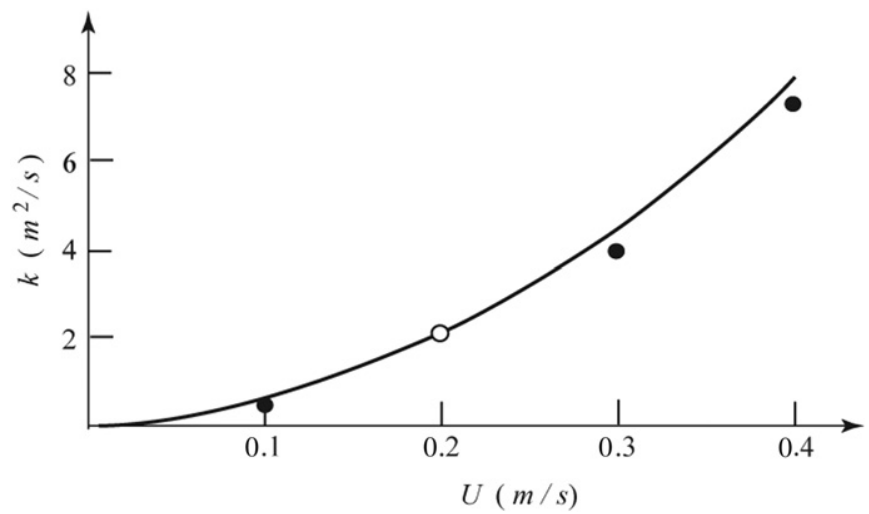

Fig. 6. The equilibrium shear diffusivity diagnosed from the numerical solution (circles) plotted against the baroclinic amplitude $U$. The curve going through the control case (open circle) represents the quadratic dependence. 


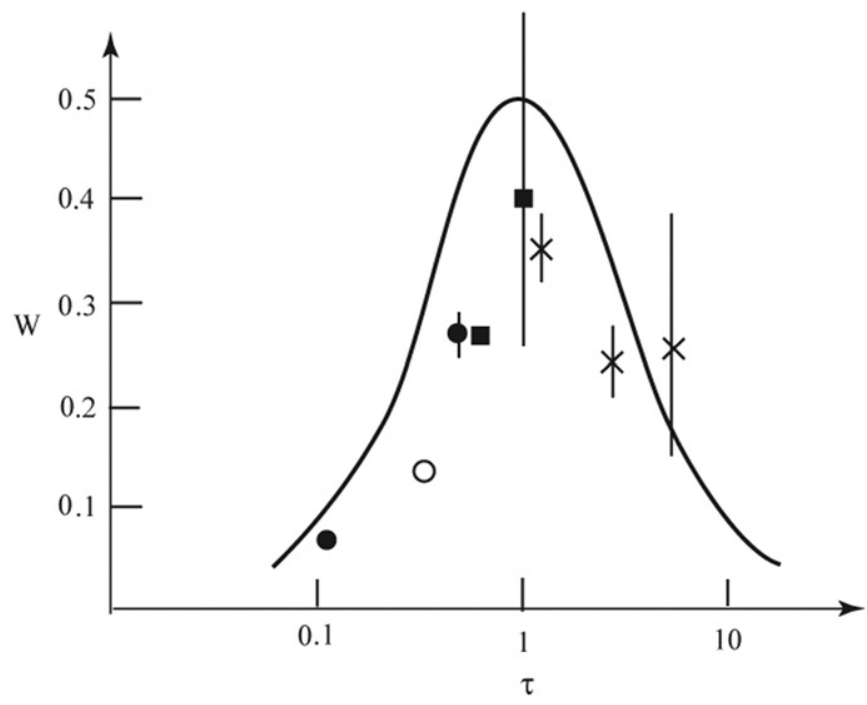

Fig. 7. The analytical diffusivity window (solid curve) and the values diagnosed from the numerical solution. The open circle is the control case of $h=16 \mathrm{~m}$, forcing period of $0.5 \mathrm{~d}$ and $v=10^{-3} \mathrm{~m}^{2} \mathrm{~s}^{-1}$; and moving from left to right, the solid circles are for additional thermocline thickness of 24 and $12 \mathrm{~m}$; the solid squares are for additional forcing periods of 1 and $2 \mathrm{~d}$; and the crosses are for the above three forcing periods but using a vertical diffusivity of $5 \times 10^{-3} \mathrm{~m}^{2} \mathrm{~s}^{-1}$. Also indicated are the error bars (not shown when indistinguishable from the markers).

\section{Application}

With the validation of the diffusivity window by the numerical calculations, we shall next apply the model derivation to the actual coastal environment. Given the crudeness of the model, it provides only an order-of-magnitude estimate of the shear diffusivity, which nonetheless suffices in assessing whether the shear dispersion may account for the observed phenomena.

Among the model parameters, the vertical diffusivity $v$ is the least certain, which may span over three orders of magnitude (Gregg et al., 1999): Over the quiescent mid-shelf, it has the openocean value of $O\left(10^{-5} \mathrm{~m}^{2} \mathrm{~s}^{-1}\right)$ (MacKinnon and Gregg, 2003b; Oakey and Greenan, 2004; Ledwell et al., 2004), but over the outer shelf where internal tides are prominent, it attains a magnitude of $O\left(10^{-4}-10^{-3} \mathrm{~m}^{2} \mathrm{~s}^{-1}\right)$ (Inall et al., 2000; Sharples et al., 2001; Rippeth and Inall, 2002; Carter et al., 2005), then at the passage of internal solibores, it may reach beyond $O\left(10^{-3} \mathrm{~m}^{2} \mathrm{~s}^{-1}\right)$ (Moum et al., 2003; MacKinnon and Gregg, 2003b). Since the saline intrusion is a feature over the outer shelf, we use the value of $5 \times 10^{-4} \mathrm{~m}^{2} \mathrm{~s}^{-1}$ estimated by Sharples et al. (2001) as a baseline value. Since this value is determined from the decay of the tidalmean baroclinic energy between two cross-shore moorings, it represents a spatial-temporal average hence more pertinent for our purpose - considering the extreme variability of this property. Furthermore, the baroclinic power spectrum shown in Fig. 2 is from the same data set, which would aid its combined use with the above diffusivity value in the later assessment of the shear dispersion. Using in addition $20 \mathrm{~m}$ as representative of the thermocline thickness (see Fig. 1), the diffusive time (12) would be $5.9 \mathrm{~d}$. Since this is much longer than the period of the semidiurnal tide, we suspect that the latter is not effective in driving the shear dispersion, as seen next.

Based on Fig. 2, we take the power density to be $20 \mathrm{~cm}^{2} \mathrm{~s}^{-2} \mathrm{~d}$ for the semi-diurnal tide, then with a record length of $13 \mathrm{~d}$ and 10 degrees of freedom (hence a bandwidth of $5 / 13 \approx 0.38 \mathrm{~d}^{-1}$ ), the baroclinic amplitude is estimated from (32) to be $U \approx(8 \times 20 \times$ $0.38)^{1 / 2} \approx 7.8 \mathrm{~cm} \mathrm{~s}^{-1}$. Since the internal tides are highly episodic, the above amplitude represents an average over the record length even though the observed amplitude can at times far exceed this value. With the aforementioned parameter values, we calculate the equilibrium shear diffusivity to be $0.22 \mathrm{~m}^{2} \mathrm{~s}^{-1}$ based on the analytical formulae (21) and (23), and if we use the smaller midshelf vertical diffusivity, the diffusive time would be longer, rendering even smaller shear diffusivity, which thus falls far short of its observed value of $O\left(10 \mathrm{~m}^{2} \mathrm{~s}^{-1}\right)$ (Section 2$)$.

Although the record length for Fig. 2 is too short to accurately gage the low-frequency power density (say, longer than $10 \mathrm{~d}$ ), one may assume it to be essentially white with a value of $40 \mathrm{~cm}^{2} \mathrm{~s}^{-2} \mathrm{~d}$. This would yield a baroclinic amplitude of $5.7 \mathrm{~cm} \mathrm{~s}^{-1}$, not much different from that of the semi-diurnal tide, but applying the analytical formula, the equilibrium shear diffusivity would be $12.1 \mathrm{~m}^{2} \mathrm{~s}^{-1}$, which is more than 50 times greater than that induced by the semi-diurnal tide. This dramatic increase stems from the steep rise of the shear diffusivity (23) when the forcing period exceeds the diffusive time (estimated above to be several days). But as cautioned earlier (Section 4), although the low-frequency forcing propels a stronger shear dispersion over the long haul (the above estimate pertains to the equilibrium shear diffusivity), this effectiveness may not be reflected in the short-term tracer dispersal, which in fact may undergo more prolonged - hence more severe - contraction associated with the low-frequency forcing (see Fig. 4). In other words, even subjected to similar low-frequency forcings, tracer patches tracked over several days may exhibit any value (even negative) of the shear diffusivity below about $10 \mathrm{~m}^{2} \mathrm{~s}^{-1}$ without contradicting its high equilibrium value - a factor that one must keep in mind in interpreting the tracer data.

The above estimates compare the efficiency of the semidiurnal versus the low-frequency forcing in driving the shear dispersion, but for a broadband continuum shown in Fig. 2, we need to apply the spectral representation in estimating the longterm (canonical) shear diffusivity. The diffusivity window shown in the figure (the thick dashed line) corresponds incidentally to the vertical diffusivity used above $\left(5 \times 10^{-4} \mathrm{~m}^{2} \mathrm{~s}^{-1}\right)$, which is seen to fall within the low-frequency plateau of the spectrum. Marking this plateau by the dotted line denoted as $\Gamma_{0}$, the spectral integration (28) becomes

$$
\begin{aligned}
k_{\text {can }} & \approx \frac{\Gamma_{0}}{4 \pi} \int_{0}^{\infty} W \mathrm{~d}(\ln \sigma) \\
& =\frac{\Gamma_{0}}{8},
\end{aligned}
$$

a particularly simple expression, which contains no dependence on the vertical diffusivity. That is, for the nominal condition of long diffusive time and - hence - relatively flat power spectrum encompassing the diffusivity window, the canonical shear diffusivity is simply one eighth of this spectral density.

Setting $\Gamma_{0} \approx 20 \mathrm{~cm}^{2} \mathrm{~s}^{-2} \mathrm{~d}$, (35) yields $k_{\text {can }} \approx 21.6 \mathrm{~m}^{2} \mathrm{~s}^{-1}$, which is of the same order-of-magnitude as its observed value noted in Section 2. Being the canonical shear diffusivity, it is the value that is more pertinent for assessing the long-time dispersion, such as the saline intrusion through the stratified season. Since the intruding distance can be equated with the square root of the second moment given in (20), it would be about $18 \mathrm{~km}$ after three months. This is sufficient to account for the observed intrusion shown in Fig. 1, which represents the mid-September condition hence several months after the late-spring emergence of the seasonal thermocline.

The saline intrusion reaches its maximum extent at the end of the stratified season when the shear dispersion ceases, but there could be other limitations on its intrusion distance. Over the MidAtlantic Bight of the North America, for example, there is a mean alongshore flow toward the south, so the downstream distance would encode the time progression of the saline intrusion - the reason that the observed feature becomes more prevalent 
southward toward Cape Hatteras (Lentz, 2003); and if the transit time through the shelf is shorter than the stratified season, it would curtail the intruding distance. Because of this constraint in addition to the regional difference in the efficacy of the shear dispersion, the saline intrusion need not be a common feature among various shelves and its observed prominence in the MidAtlantic Bight may very well be related to its length hence the long transit time.

\section{Summary and discussion}

Through an idealized analytical model, we consider the rectified shear dispersion in the thermocline induced by the baroclinic motion. For a monochromatic forcing, the instantaneous shear diffusivity would vacillate, but its time-mean is always positive and stabilizes over time long compared with both the forcing period and the diffusive time. This "equilibrium" shear diffusivity is negligible for short-period forcing but rises sharply when the forcing period transition through the diffusive time and levels off for still longer-period forcing. Since the diffusive time is generally much longer than the period of internal tides, the latter are ineffective in driving the shear dispersion, which is thus propelled mainly by the low-frequency baroclinic motion. As such, the short-term evolution of the tracer patch would exhibit highly varied behavior - even contraction - and provides a poor gage for the effectiveness of the long-term dispersion.

Since the baroclinic motion over the shelf is inherently broadband, one needs a spectral representation of the shear diffusivity to assess its long-term value. This "canonical" shear diffusivity is found to be simply the baroclinic power band-passed by a diffusivity window on the log-frequency space, which thus may be readily assessed given the regional forcing and mixing environment. Since in a shelf environment, the low-frequency baroclinic power spectrum is relatively flat within the nominal diffusivity window, it is further derived that the canonical shear diffusivity is simply one-eighth of this plateau - a surprisingly simple result that is independent of the vertical diffusivity. When applied to the seasonal thermocline of the Mid-Atlantic shelf, the long-term shear diffusivity is about $20 \mathrm{~m}^{2} \mathrm{~s}^{-1}$, which is sufficient to account for the observed tracer dispersal and saline intrusion in the thermocline.

The analytical model has employed many simplifying assumptions that cannot be strictly justified for the observed phenomena. Among the more serious is the use of constant vertical diffusivity in the thermocline whereas the observed diapycnal mixing is highly episodic, which may interact with the variable shear to produce other rectified effect. This problem is partly ameliorated by the finding that the shear dispersion is propelled by the low-frequency baroclinic motion so the random phase of the diapycnal mixing would be smoothed out. It is in fact based on this premise that in the analytical model the vertical mixing may be treated as a regional property separable from the baroclinic forcing field. The analytical derivation also assumes the tracer displacement to be small compared with the lateral scale of the mean tracer concentration so that the tracer perturbation is governed by the linearized equation. While this imposes a rather stringent condition, which is certainly not met in some numerical experiments, the latter nonetheless have validated the analytically derived shear diffusivity, suggesting its wider applicability than the model assumptions would indicate.

Because of the technical constraints on the numerical integration, we have applied model parameters directly to test the analytically derived shear diffusivity. This approach is justified in that the primary inquiry is the effectiveness of the shear dispersion - not the physics that governs these "external" parameters. Because of these specifications, on the other hand, the numerical solution does not constitute a simulation of the observed phenomena, least of which the saline intrusion as is obvious in our use of a vertical salinity strip. The model findings on the other hand have underscored additional difficulties that would confront attempts to simulate the saline intrusion: the most serious perhaps is the dominance of the shear dispersion by the low-frequency baroclinic motion, which may stem from nonlinear interaction of the internal tides; and to carry out the long integration needed, one may have to incorporate genesis of the thermocline to prevent its diffusive drift.

Although we consider the shear dispersion in the seasonal thermocline, the basic physics should be operative in the main thermocline where the shear may be associated with baroclinic eddies. In the subtropical North Atlantic, the tracer data of Robbins et al. (2000) show strong ventilation of the lower thermocline water, which however cannot be explained by the subduction; they found instead it requires isopycnal diffusivity of $O\left(10^{3} \mathrm{~m}^{2} \mathrm{~s}^{-1}\right)$, the source of which remains unknown. To show that the shear dispersion can produce such large diffusivity, we assume a thermocline thickness of $30 \mathrm{~m}$ and diapycnal diffusivity of $10^{-4} \mathrm{~m}^{2} \mathrm{~s}^{-1}$ (Munk and Wunsch, 1998) to yield a diffusive timescale of $73 \mathrm{~d}$. Assuming similar timescale for the baroclinic eddies and baroclinic amplitude of $0.2 \mathrm{~m} \mathrm{~s}^{-1}$, the analytical formulae would yield a shear diffusivity of $1.3 \times 10^{3} \mathrm{~m}^{2} \mathrm{~s}^{-1}$, not unlike that required. It is recognized that the shear dispersion is propelled in the direction of the shearing motion, but with eddy motion that transition through all directions, the above order-ofmagnitude estimate remains justified. This deduction underscores the point that it is not the vigor of the vertical mixing but the commensurability of the diffusive and forcing timescales that determines the effectiveness of the shear dispersion. It is the perceived tidal dominance of the shear that has stipulated the need for large vertical diffusivity, but with the presence of comparable low-frequency shear, such strong vertical mixing is no longer necessary. In fact, in the extreme case of a steady shear, the lateral diffusivity would increase indefinitely with decreasing vertical diffusivity (Taylor, 1953) - though it also takes longer time to attain the equilibrium value.

Since the shear dispersion that drives the saline intrusion also enhances the offshore transport of coastal-derived pollutants, it is important for practical applications to parameterize the process in regional models that do not resolve internal tides or small scale vertical mixing. And since under nominal coastal condition, we derive that the shear diffusivity can be approximated by (35), the latter provides a crude yet easily applied parameterization scheme. It is recognized that shear dispersion is likely more effective in the benthic layer, driven by the barotropic tides and their attendant strong vertical mixing; but since these processes are more easily resolved in regional models, there is less need for their parameterization.

\section{Acknowledgments}

The authors want to thank the anomalous reviewers for their constructive comments, and Jonathan Sharples and Derek Barrage for their help with Figs. 1 and 2 respectively taken from their published papers. Dake Chen is supported in part by grants from the Ministry of Science and Technology (2010DFA21012), the State Oceanic Administration (201105018), and the National Science Foundation (91128204) of China.

\section{Appendix. Shear diffusivity}

For a monochromatic forcing, we have

$\hat{u}=\frac{1}{2} U \cos (2 \pi \omega t+\theta)$, 
where $U$ is the baroclinic amplitude and the phase $\theta$ pertains to the timing difference between the tracer release and the peak positive baroclinic current. Substituting (A.1) and (16) into (15), we derive

$$
\begin{aligned}
k / k_{\mathrm{eq}}= & 2 \cos (2 \pi \omega t+\theta)\left[\cos (2 \pi \omega t+\theta)+\frac{1}{\tau} \sin (2 \pi \omega t+\theta)\right. \\
& \left.-\exp (-\alpha t)\left(\cos \theta+\frac{1}{\tau} \sin \theta\right)\right]
\end{aligned}
$$

where the shear diffusivity $k$ has been non-dimensionalized by (21) and $k_{\text {eq }}$ is the equilibrium shear diffusivity given by (23). The average of (A.2) over the time interval $[0, t]$ yields

$$
\begin{aligned}
\bar{k} / k_{\text {eq }}= & +\frac{1}{4 \pi \omega t}[\sin 2(2 \pi \omega t+\theta)-\sin 2 \theta] \\
& -\frac{1}{2 \alpha t}[\cos 2(2 \pi \omega t+\theta)-\cos 2 \theta] \\
& +\frac{32 W}{2 \pi \omega t}\left(\cos \theta+\frac{1}{\tau} \sin \theta\right)\{\exp (-\alpha t)[\cos (2 \pi \omega t+\theta) \\
& \left.\left.-\frac{1}{\tau} \sin (2 \pi \omega t+\theta)\right]-\left(\cos \theta-\frac{1}{\tau} \sin \theta\right)\right\}
\end{aligned}
$$

where $W$ is the diffusivity window given in (31). The timeintegrated shear diffusivity is (A.3) multiplied by $t$. It is seen that the large-time limit of (A.3) yields

$\bar{k} \rightarrow k_{\mathrm{eq}}$ as $t \rightarrow \infty$,

as expected.

\section{References}

Aikman, F., 1984. Pycnocline development and its consequences in the Middle Atlantic Bight. Journal of Geophysical Research 89, 685-694.

Baines, P.G., 1982. On internal tide generation models. Deep-Sea Research 29 (3A), 307-338.

Boicourt, W.C., Hacker, P.W., 1976. Circulation on the Atlantic continental shelf of the United States, Cape May to Cape Hatteras. Memoires de la Societd Royale des Sciences des Liege 6e (10), 187-200.

Burrage, D.M., Garvine, R.W., 1988. Summertime hydrography at the shelfbreak front in the Middle Atlantic Bight. Journal of Physical Oceanography 18 , 1309-1319.

Carter, G.S., Gregg, M., Lien, R.-C., 2005. Internal waves, solitary waves, and mixing on the Monterey Bay shelf. Continental Shelf Research 25, 1499-1520.

Chen, D., Ou, H.W., Dong, C., 2003. A model study of internal tides in coastal frontal zone. Journal of Physical Oceanography 33, 170-187.

Churchill, J.H., Manning, J.P., Beardsley, R.C., 2003. Slope water intrusions onto Georges Bank. Journal of Geophysical Research 108 (C11), 8012, http://dx.doi.org http://dx.doi.org/10.1029/2002JC001400.

Colosi, J.A., Beardsley, R.C., Lynch, J.F., Gawarkiewicz, G., Chiu, C.-S., Scotti, A., 2001 Observations of nonlinear internal waves on the outer New England continental shelf during the summer Shelfbreak Primer study. Journal of Geophysical Research 106, 9587-9601.

Flagg, C.N., Houghton, R.W., Pietrafesa, L.J., 1994. Summertime thermocline salinity maximum intrusions in the Mid-Atlantic Bight. Deep-Sea Research, II $41,325-340$.

Gordon, A.L., Aikman III, F., 1981. Salinity maximum in the pycnocline of the Middle Atlantic Bight. Limnology and Oceanography 26 (1), 123-130.

Gregg, M.C., Winkel, D.P., MacKinnon, J.A., Lien, R.-C., 1999. Mixing over shelves and slopes. In: Dynamics of Oceanic Internal Gravity Waves II, Proceedings, Muller, P., Henderson, D. (Eds.), Hawaiian Winter Workshop, January 18-22, 1999. University of Hawaii at Manoa, pp. 35-42.

Horne, E.P.W., 1978. Interleaving at the subsurface front in the slope water off Nova Scotia. Journal of Geophysical Research 83, 3659-3671.

Houghton, R.W., 1997. Lagrangian flow at the foot of a shelfbreak front using a dye tracer injected into the bottom boundary layer. Geophysical Reserch Letters 24, 2035-2038.

Houghton, R.W., Marra, J., 1983. Physical/biological structure and exchange across the thermohaline shelf/slope front in the New York Bight. Journal of Geophysical Research 88 (C7), 4467-4481.
Inall, M.E., Rippeth, T.P., Sherwin, T.J., 2000. Impact of nonlinear waves on the dissipation of internal tidal energy at a shelf break. Journal of Geophysical Research 105 (C4), 8687-8705.

Jenkins, G.M., Watts, D.G., 1968. Spectral Analysis and Its Applications. Holden-day $525 \mathrm{pp}$.

Ledwell, J.R., Duda, T.F., Sundermeyer, M.A., Seim, H.E., 2004. Mixing in a coastal environment. Part I: A view from dye dispersion. Jornal of Geophysical Research 109 (C10), C10013, http://dx.doi.org/http://dx.doi.org/10.1029/ 2003JC002194.

Ledwell, J.R., Watson, A.J., Law, C.S., 1993. Evidence for slow mixing across the pycnocline from an open-ocean tracer-release experiment. Nature 364, 701-703.

Ledwell, J.R., Watson, A.J., Law, C.S., 1998. Mixing of a tracer in the pycnocline. Journal of Geophysical Research 103 (C10), 21,499-21,529.

Lentz, S.J., 2003. A climatology of salty intrusions over the continental shelf from Georges Bank to Cape Hatteras. Journal of Geophysical Research 108 (C10), 3326, http://dx.doi.org/http://dx.doi.org/10.1029/2003JC001859.

Liu, A.K., Chang, Y.S., Hsu, M.-K., Liang, N.K., 1998. Evolution of nonlinear internal waves in the East and South China Seas. Journal of Geophysical Research 103. 7995-8008.

MacKinnon, J.A., Gregg, M.C., 2003a. Shear and baroclinic energy flux on the summer New England shelf. Journal of Physical Oceanography 33, 1462-1475.

Mackinnon, J.A. Gregg, M.C., 2003b. Mixing on the late-summer New England shelf - solibores, shear, and stratification. Journal of Physical Oceanography 33, 1476-1492.

Moum, J.N., Farmer, D.M., Smyth, W.D., Armi, L., Vagle, S., 2003. Structure and generation of turbulence at interfaces strained by internal solitary waves propagating shoreward over the continental shelf. Journal of Physical Oceanography 33 (10), 2093-2112.

Munk, W., Wunsch, C., 1998. Abyssal recipes II: energetics of tidal and wind mixing. Deep-Sea Research I 45, 1977-2010.

Oakey, N.S., Greenan, B.J.W., 2004. Mixing in a coastal environment. Part II: A view from microstructure measurements. Journal of Geophysical Research 109 (C10), C10014, http://dx.doi.org/http://dx.doi.org/10.1029/2003JC002193.

Okubo, A., 1967. The effect of shear in an oscillatory current on horizontal diffusion from an instantaneous source. International Journal of Oceanology and Limnology 1, 194-204.

Ou, H.W. Maas, L., 1986. Tidal-induced buoyancy flux and mean transverse circulation. Continental Shelf Research 5, 611-628.

Palmer, M.R., Rippeth, T.P., Simpson, J.H., 2008. An investigation of internal mixing in a seasonally stratified shelf sea. Journal of Geophysical Research 113 C12005, http://dx.doi.org/http://dx.doi.org/10.1029/2007JC004531.

Rippeth, T.P., Inall, M.E., 2002. Observations of the internal tide and associated mixing across the Malin shelf. Journal Geophysical Research 107, 3028, http:// dx.doi.org/http://dx.doi.org/10.1029/2000JC000761.

Robbins, P.E., Price, J.F., Owens, W.B., Jenkins, W.J., 2000. The importance of lateral diffusion for the ventilation of the lower thermocline in the subtropical North Atlantic. Journal of Physical Oceanography 30, 67-89.

Saffman, P.G., 1962. The effect of wind shear on horizontal spread from an instantaneous ground source. Quarterly Journal of the Royal Meteorological Society $88,382-393$.

Sharples, J., Moore, C.M., Abraham, E.R., 2001. Internal tide dissipation, mixing, and vertical nitrate flux at the shelf edge of NE New Zealand. Journal of Geophysical Research 106 (C7), 14069-14081.

Smith, R., 1982. Contaminant dispersion in oscillatory flows. Journal of Fluid Mechanics 114, 379-398.

Sundermeyer, M.A., Lelong, M.-P., 2005. Numerical simulations of lateral dispersion by the relaxation of diapycnal mixing events. Journal of Physical Oceanography 35, 2368-2386.

Sundermeyer, M.A., Ledwell, J.R., 2001. Lateral dispersion over the continental shelf: Analysis of dye release experiments. Journal of Geophysical Research 106 (C5), 9603-9621.

Sundermeyer, M.A., Ledwell, J.R., Oakey, N.S., Greenan, B.J.W., 2005. Stirring by small-scale vortices caused by patchy mixing. Journal of Physical Oceanography $35,1245-1262$.

Taylor, G.I., 1953. Dispersion of soluble matter in solvent flowing slowly through a tube. Proceedings of the Royal Society of London, Series A 219, 186-203.

Wang, D.-P., 1982. Development of a three-dimensional limited-area (island) shelf circulation model. Journal of Physical Oceanography 12, 605-617.

Welch, C.S., 1981. Mid-level intrusions at the continental shelf edge. Journal of Geophysical Research 86, 11,013-11,019.

Young, W.R., Rhines, P.B., Garrett, C.J.R., 1982. Shear flow dispersion, interna waves and horizontal mixing in the ocean. Journal of Physical Oceanography $12,515-527$.

Zimmerman, J.T.F., 1986. The tidal whirlpool: a review of horizontal dispersion by tidal and residual currents. Netherlands Journal of Sea Research 20, 133-154. 